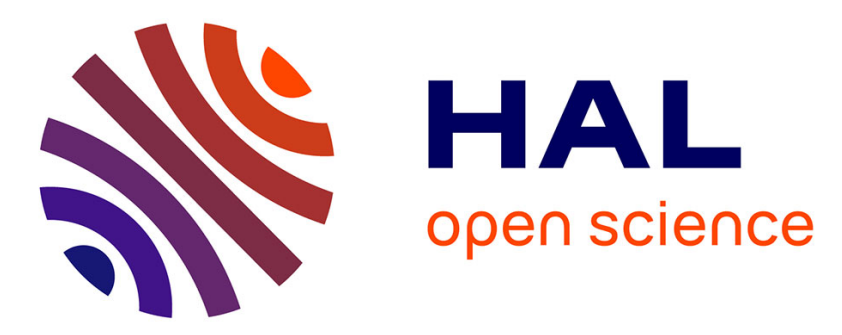

\title{
Black-Box Optimization Benchmarking the IPOP-CMA-ES on the Noisy Testbed
}

\author{
Raymond Ros
}

\section{To cite this version:}

Raymond Ros. Black-Box Optimization Benchmarking the IPOP-CMA-ES on the Noisy Testbed. Genetic and Evolutionary Computation Conference 2010, Jul 2010, Portland, OR, United States. inria-00473778

\section{HAL Id: inria-00473778 \\ https://hal.inria.fr/inria-00473778}

Submitted on 16 Apr 2010

HAL is a multi-disciplinary open access archive for the deposit and dissemination of scientific research documents, whether they are published or not. The documents may come from teaching and research institutions in France or abroad, or from public or private research centers.
L'archive ouverte pluridisciplinaire HAL, est destinée au dépôt et à la diffusion de documents scientifiques de niveau recherche, publiés ou non, émanant des établissements d'enseignement et de recherche français ou étrangers, des laboratoires publics ou privés. 


\title{
Black-Box Optimization Benchmarking the IPOP-CMA-ES on the Noisy Testbed
}

\author{
Comparison to the BIPOP-CMA-ES
}

\author{
Raymond Ros \\ TAO Team-Project - INRIA Saclay \\ LRI, Bat 490, Univ. Paris-Sud \\ F-91405 Orsay Cedex, France \\ raymond.ros@inria.fr
}

\begin{abstract}
We benchmark the IPOP-CMA-ES on the noisy testbed of the BBOB 2010 workshop. The performances of the IPOPCMA-ES are compared to those of the BIPOP-CMA-ES. Both algorithms are shown to perform comparably on the BBOB noisy testbed.
\end{abstract}

\section{Categories and Subject Descriptors}

G.1.6 [Numerical Analysis]: Optimization-global optimization, unconstrained optimization; F.2.1 [Analysis of Algorithms and Problem Complexity]: Numerical Algorithms and Problems

\section{General Terms}

Algorithms

\section{Keywords}

Benchmarking, Black-box optimization, Evolution strategies

\section{ALGORITHM PRESENTATION}

The algorithm Covariance Matrix Adaptation-Evolution Strategy (CMA-ES) [9] is a stochastic search method based on a population. We choose to apply the $\left(\mu / \mu_{w}, \lambda\right)$-CMA$\mathrm{ES}[3,7,8]$ in this paper. The Increasing POPulation-size (IPOP) restart policy was proposed for the CMA-ES in [1]. The resulting IPOP-CMA-ES algorithm uses a population doubling in size at each restarts.

We compare the performances of the IPOP-CMA-ES to those of the BIPOP-CMA-ES [4] which was proposed to the BBOB 2009 workshop. The BIPOP-CMA-ES distributes the allocated budget - number of function evaluations - between a doubling population size and a small population size policy. The BIPOP-CMA-ES showed good performances on the function testbeds of the BBOB 2009 workshop.

Permission to make digital or hard copies of all or part of this work for personal or classroom use is granted without fee provided that copies are not made or distributed for profit or commercial advantage and that copies bear this notice and the full citation on the first page. To copy otherwise, to republish, to post on servers or to redistribute to lists, requires prior specific permission and/or a fee.

GECCO'10, July 7-11, 2010, Portland, Oregon, USA

Copyright 2010 ACM 978-1-4503-0073-5/10/07 ...\$10.00.

\section{EXPERIMENTAL PROCEDURE}

The IPOP-CMA-ES was tested using the same experimental set-up as that of the BIPOP-CMA-ES [4] tested for the BBOB 2009 workshop. In particular parameter $c_{1}$ and $c_{\mu}$, terms of the learning rate $c_{\mathrm{cov}}$ are set to one fifth of the values that used for the noiseless testbed.

The only difference with the BIPOP-CMA-ES is that all the budget in terms of number of function evaluations is allocated to the doubling population size policy.

The crafting effort for IPOP-CMA-ES [5] computes to $\mathrm{CrE}=0$.

\section{RESULTS}

Results of the CPU timing experiment are given in the paper benchmarking IPOP-CMA-ES on the noiseless testbed.

Results from experiments according to [5] on the benchmark functions given in $[2,6]$ are presented in Figures 1, 2, 3 and 4 and in Tables 1 and 2 . The expected running time (ERT), used in the figures and tables, depends on a given target function value, $f_{\mathrm{t}}=f_{\mathrm{opt}}+\Delta f_{\mathrm{t}}$, and is computed over all relevant trials as the number of function evaluations executed during each trial while the best function value did not reach $f_{\mathrm{t}}$, summed over all trials and divided by the number of trials that actually reached $f_{\mathrm{t}}[5,10]$. Statistical significance is tested with the rank-sum test for a given target $\Delta f_{\mathrm{t}}\left(10^{-8}\right.$ in Figure 1) using, for each trial, either the number of needed function evaluations to reach $\Delta f_{\mathrm{t}}$ (inverted and multiplied by -1 ), or, if the target was not reached, the best $\Delta f$-value achieved, measured only up to the smallest number of overall function evaluations for any unsuccessful trial under consideration.

The performances of both IPOP-CMA-ES and BIPOPCMA-ES are pretty close with IPOP-CMA-ES being slightly faster overall but not significantly. The exception is function $f_{117}$ (Ellipsoid function with uniform noise model) in 20-D where the IPOP-CMA-ES is significantly faster but only by a factor smaller than two.

\section{REFERENCES}

[1] A. Auger and N. Hansen. A restart CMA evolution strategy with increasing population size. In Proceedings of the IEEE Congress on Evolutionary Computation (CEC 2005), pages 1769-1776. IEEE Press, 2005.

[2] S. Finck, N. Hansen, R. Ros, and A. Auger. Real-parameter black-box optimization benchmarking 

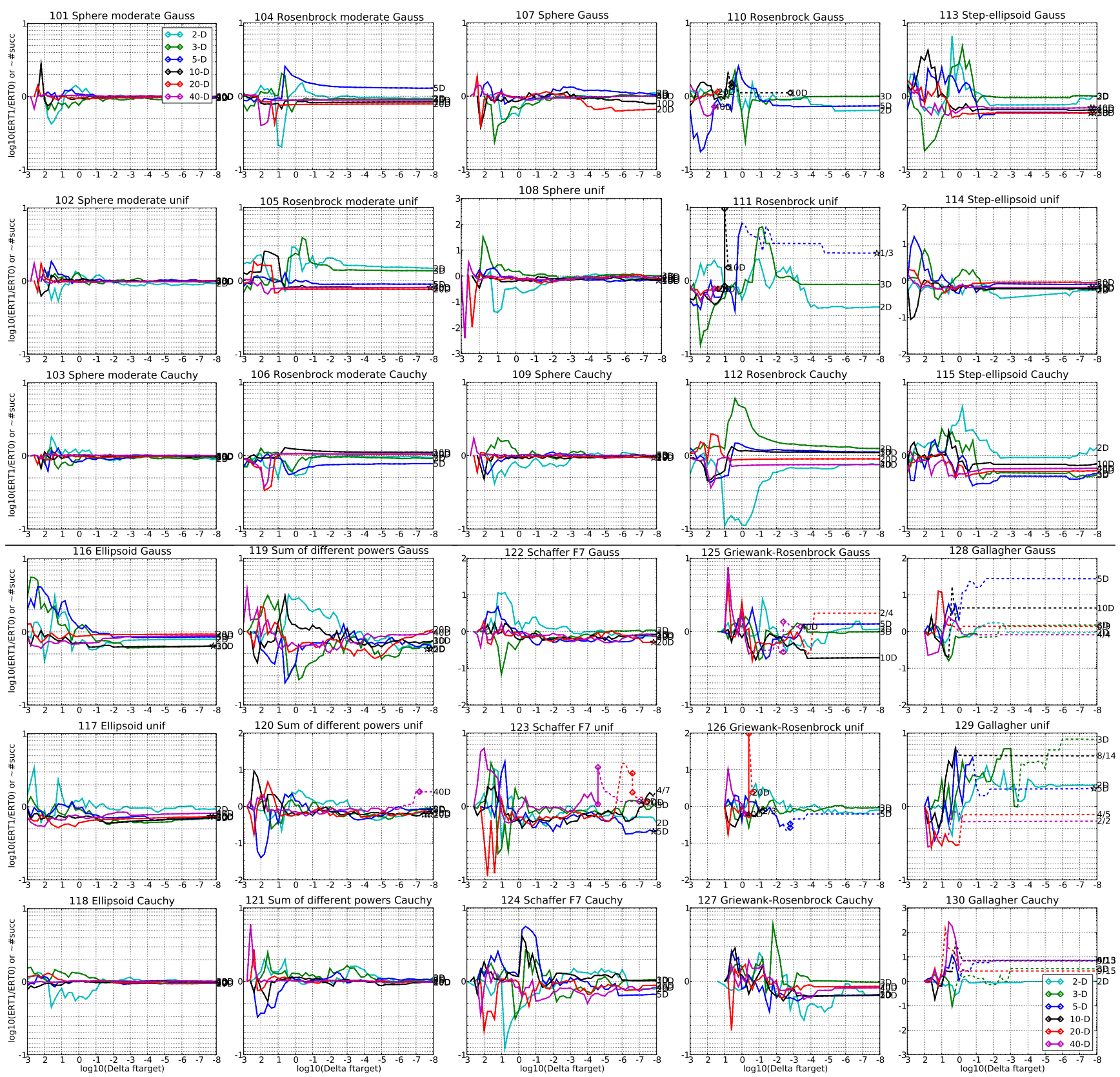

Figure 1: Ratio of the expected running times (ERT) of IPOP-CMA divided by BIPOP-CMA versus $\log _{10}(\Delta f)$ for $f_{101}-f_{130}$ in 2, 3, 5, 10, 20, 40-D. Ratios $<10^{\circ}$ indicate an advantage of IPOP-CMA, smaller values are always better. The line gets dashed when for any algorithm the ERT exceeds thrice the median of the trial-wise overall number of $f$-evaluations for the same algorithm on this function. Symbols indicate the best achieved $\Delta f$-value of one algorithm (ERT gets undefined to the right). The dashed line continues as the fraction of successful trials of the other algorithm, where 0 means $0 \%$ and the y-axis limits mean $100 \%$, values below zero for IPOP-CMA. The line ends when no algorithm reaches $\Delta f$ anymore. The number of successful trials is given, only if it was in $\{1 \ldots 9\}$ for IPOP-CMA (1st number) and non-zero for BIPOP-CMA (2nd number). Results are statistically significant with $p=0.05$ for one star and $p=10^{-\# \star}$ otherwise, with Bonferroni correction within each figure. 
101 Sphere (moderate) 104 Rosenbrock (moderate)

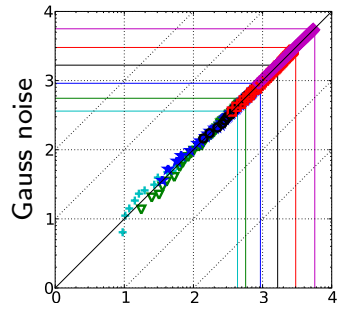

102 Sphere (moderate)

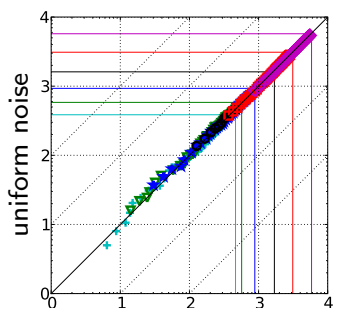

103 Sphere (moderate)

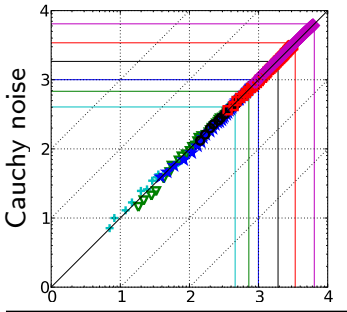

116 Ellipsoid

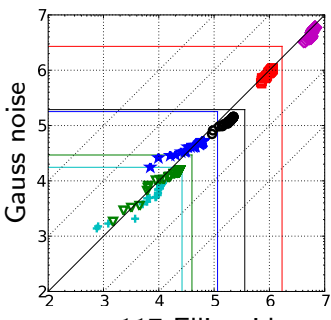

117 Ellipsoid

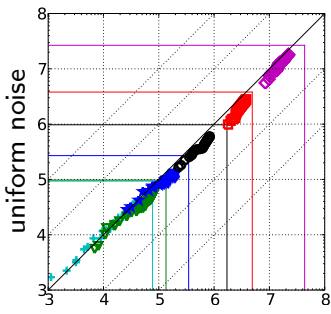

118 Ellipsoid

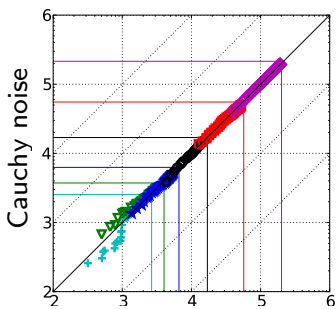

107 Sphere

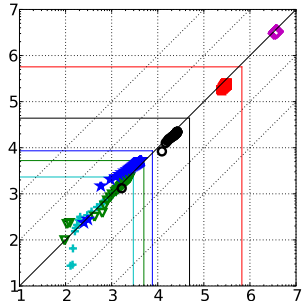

105 Rosenbrock (moderate)

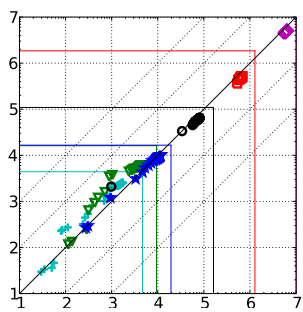

106 Rosenbrock (moderate)

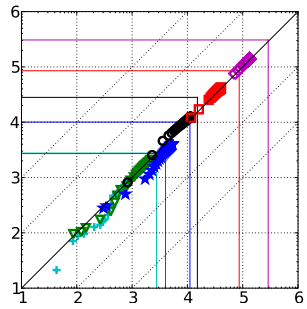

119 Sum of diff. powe

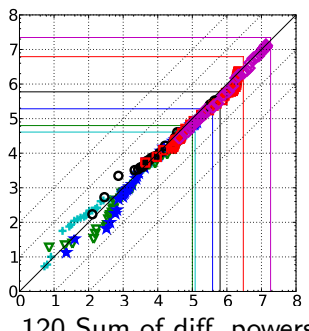

120 Sum of diff. powers

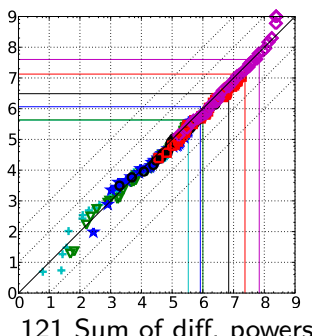

121 Sum of diff. powers

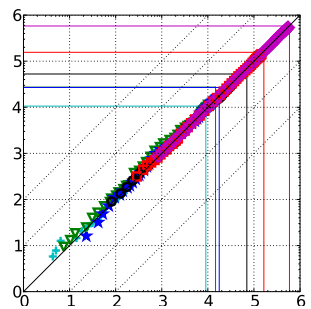

108 Sphere

109 Sphere
110 Rosenbrock
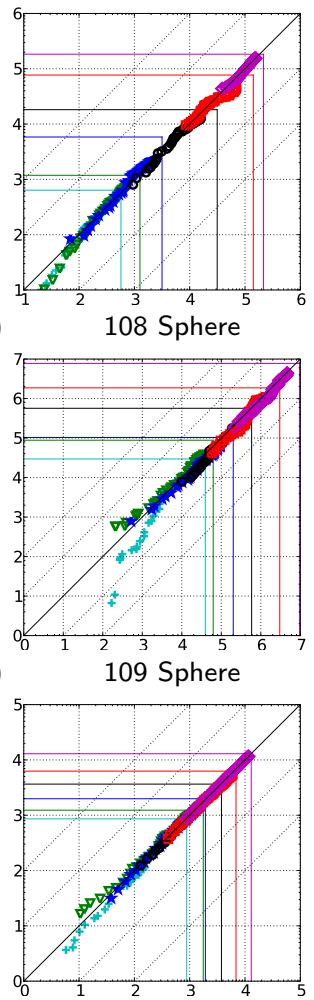

122 Schaffer F7

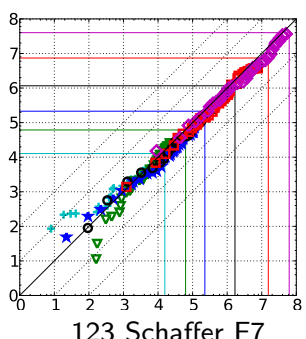

123 Schaffer F7

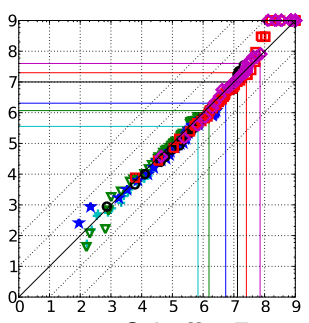

124 Schaffer F7

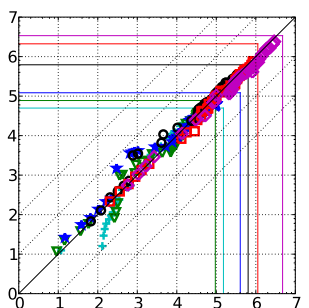

111 Rosenbrock

112 Rosenbrock
113 Step-ellipsoid
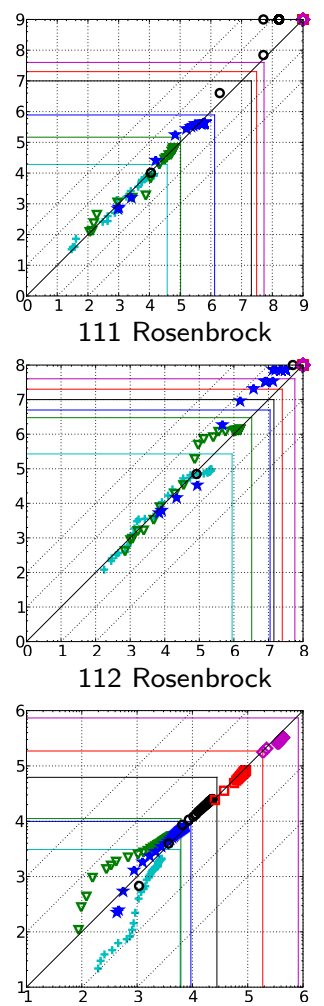

125 Griewank-Rosenbrock
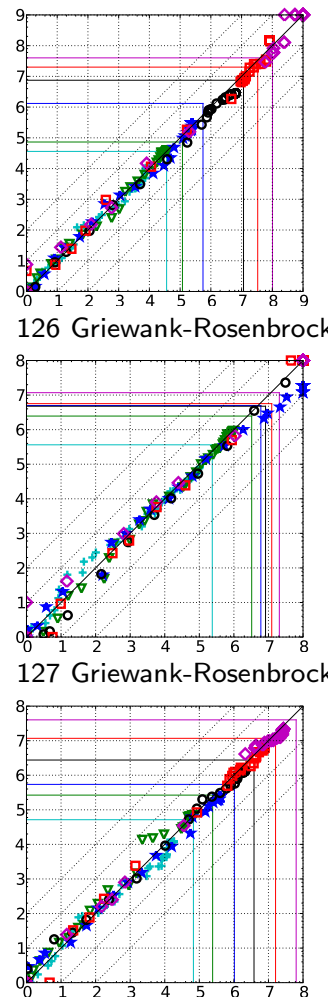

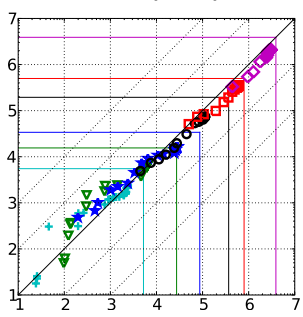

114 Step-ellipsoid

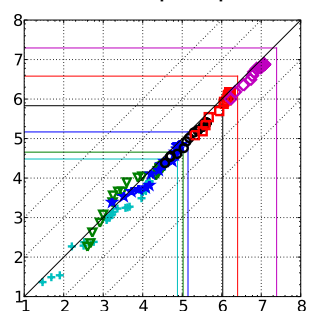

115 Step-ellipsoid

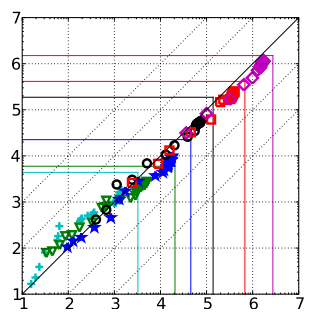

128 Gallagher

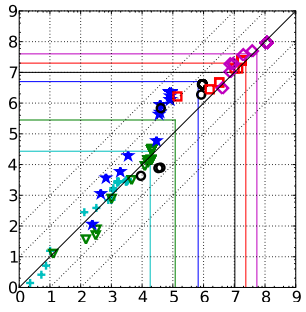

129 Gallagher

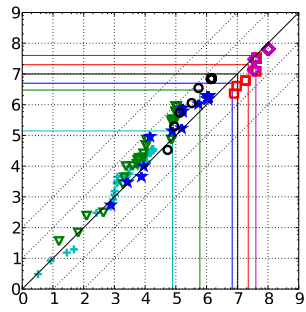

130 Gallagher

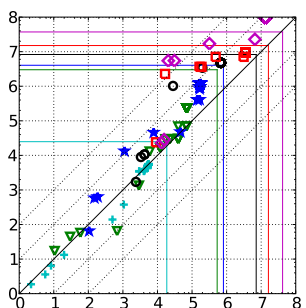

Figure 2: Expected running time (ERT in log10 of number of function evaluations) of IPOP-CMA versus BIPOP-CMA for 46 target values $\Delta f \in\left[10^{-8}, 10\right]$ in each dimension for functions $f_{101}-f_{130}$. Markers on the upper or right egde indicate that the target value was never reached by IPOP-CMA or BIPOP-CMA respectively. Markers represent dimension: 2: $+, 3: \nabla, 5: \star, 10: \circ, 20: \square, 40: \diamond$. 
$5-\mathrm{D}$
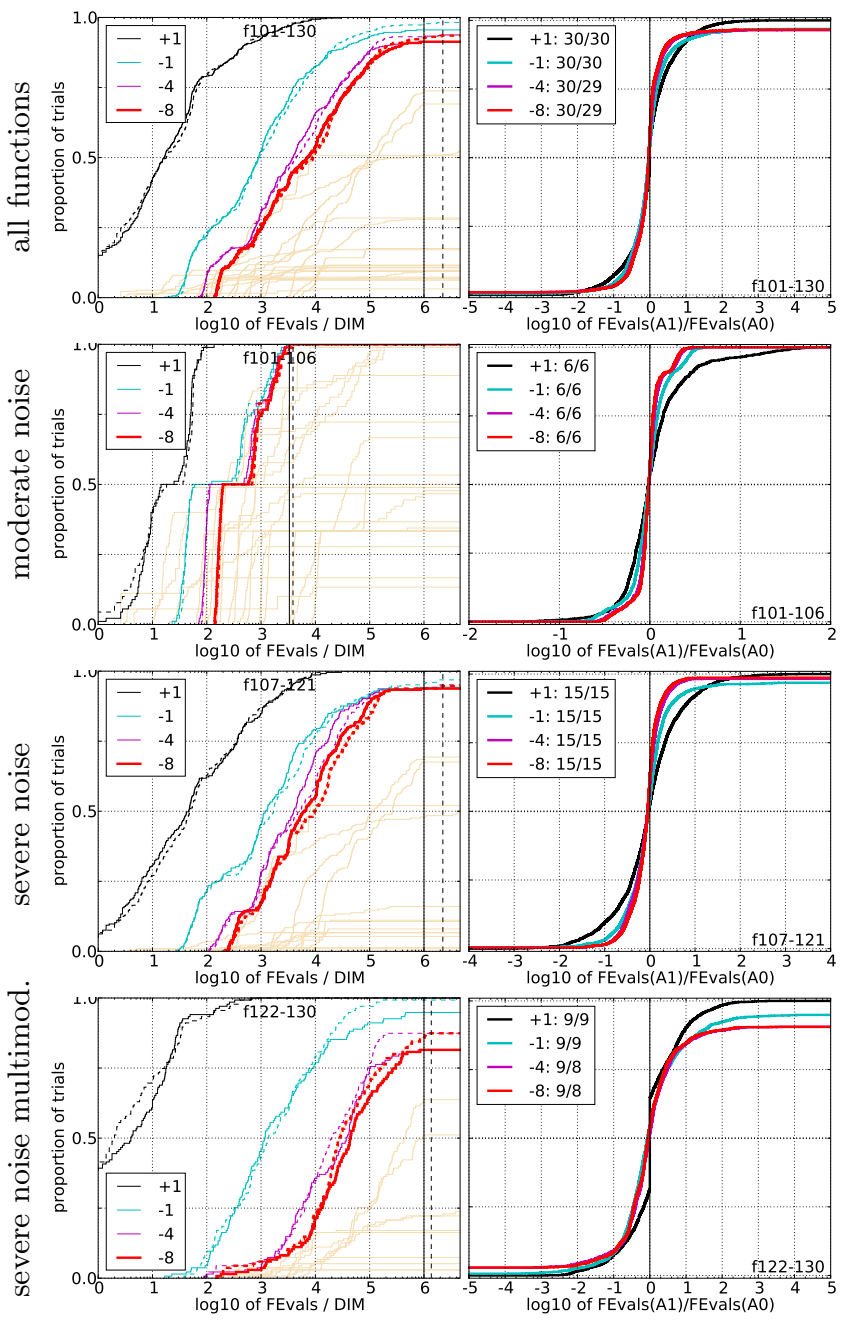

$20-\mathrm{D}$
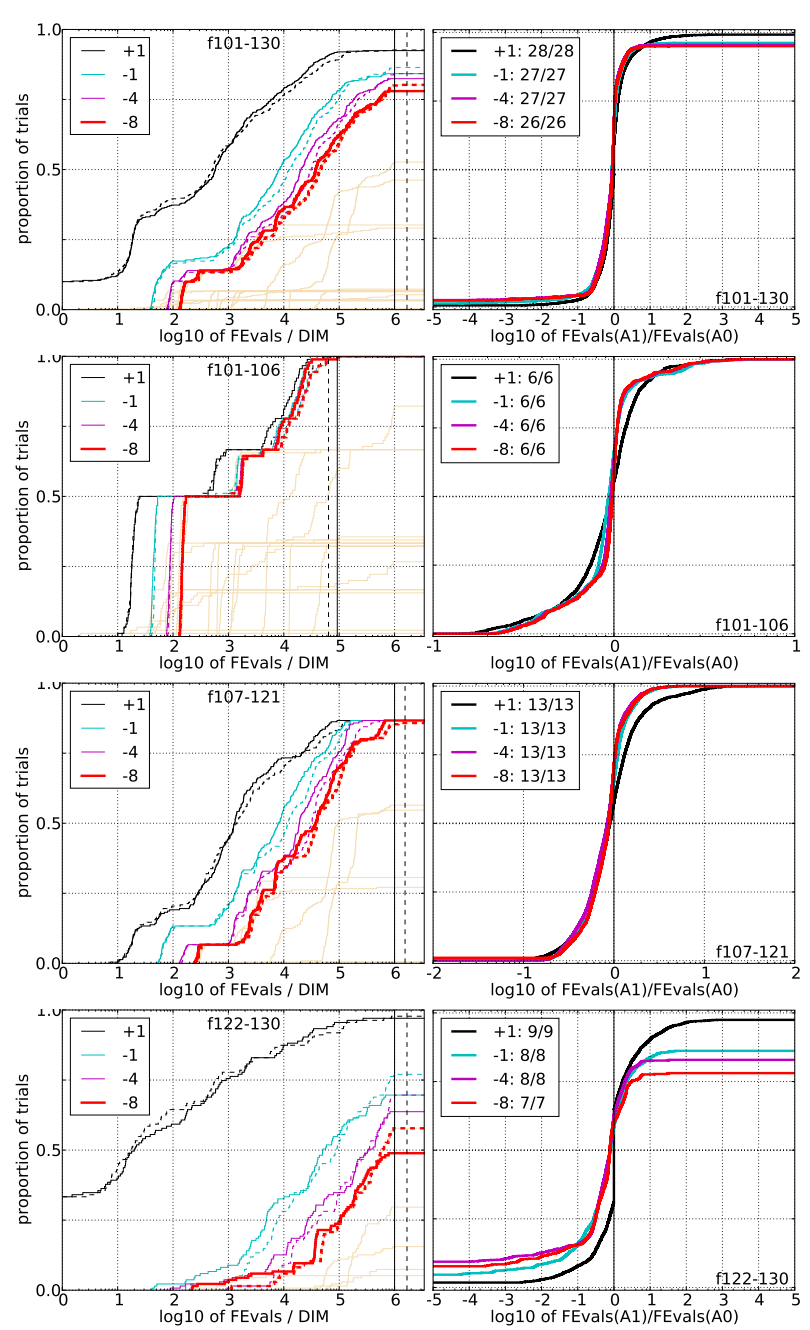

Figure 3: Empirical cumulative distributions (ECDF) of run lengths and speed-up ratios in 5-D (left) and 20-D (right). Left sub-columns: ECDF of the number of necessary function evaluations divided by dimension $D$ (FEvals/D) to reached a target value $f_{\mathrm{opt}}+\Delta f$ with $\Delta f=10^{k}$, where $k \in\{1,-1,-4,-8\}$ is given by the first value in the legend, for IPOP-CMA (solid) and BIPOP-CMA (dashed). Light beige lines show the ECDF of FEvals for target value $\Delta f=10^{-8}$ of all algorithms benchmarked during BBOB-2009. Right sub-columns: ECDF of FEval ratios of IPOP-CMA divided by BIPOP-CMA, all trial pairs for each function. Pairs where both trials failed are disregarded, pairs where one trial failed are visible in the limits being $>0$ or $<1$. The legends indicate the number of functions that were solved in at least one trial (IPOP-CMA first). 
$5-\mathrm{D}$

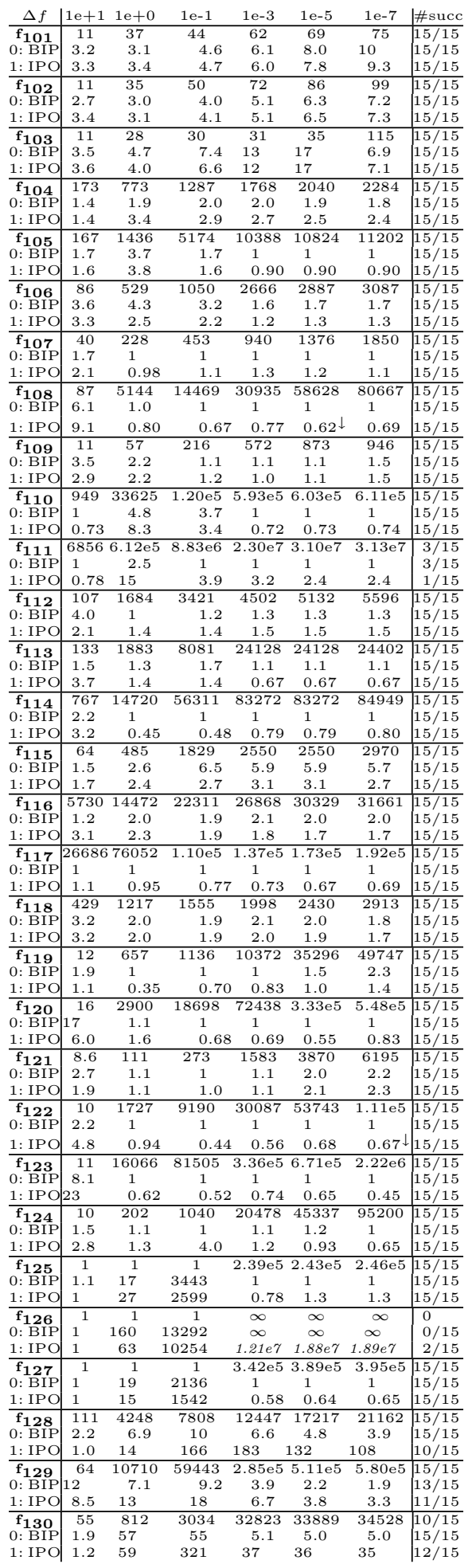

20-D

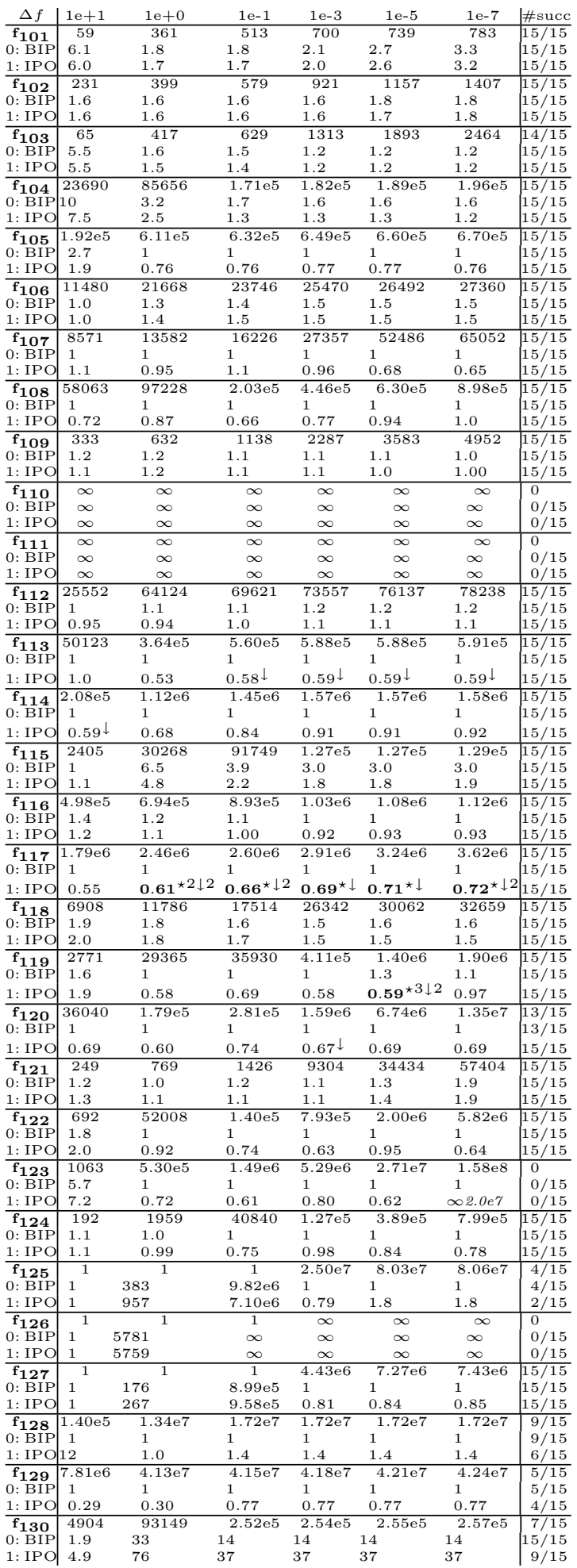

Table 1: ERT in number of function evaluations divided by the best ERT measured during BBOB-2009 (given in the respective first row) for different $\Delta f$ values for functions $f_{101}-f_{130}$. \#succ is the number of trials that reached the final target $f_{\mathrm{opt}}+10^{-8}$. 0: BIP is BIPOP-CMA and 1: IPO is IPOP-CMA. \#succ is the number of trials that reached the final target $f_{\mathrm{opt}}+10^{-8}$. 0: BIP is BIPOP-CMA and 1: IPO is IPOP-CMA. Bold entries are statistically significantly better compared to the other algorithm, with $p=0.05$ or $p=10^{-k}$ where $k>1$ is the number following the $\star$ symbol, with Bonferroni correction of 60 . 


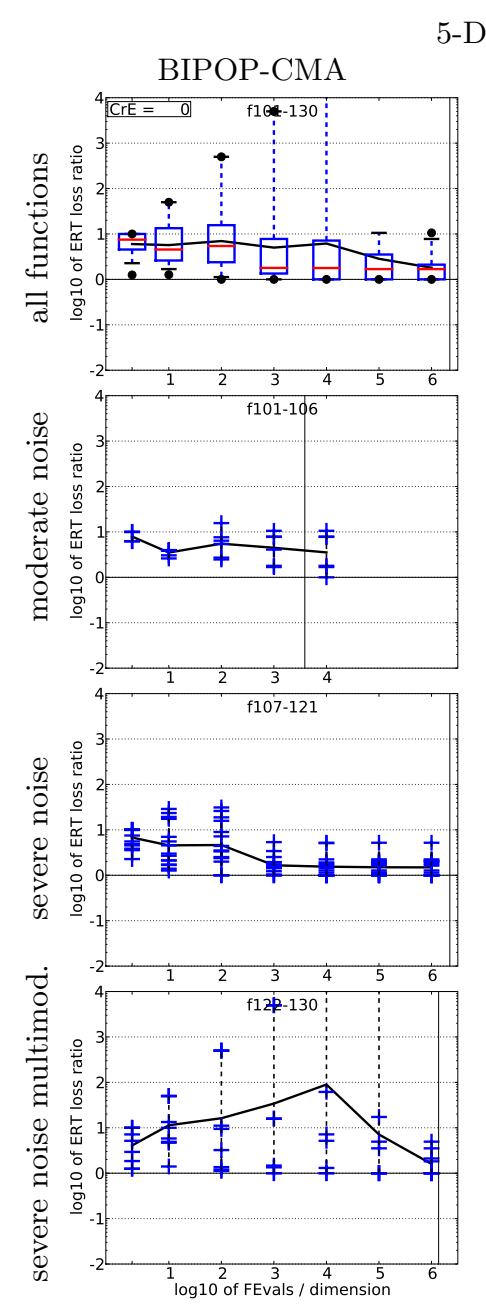

$5-\mathrm{D}$
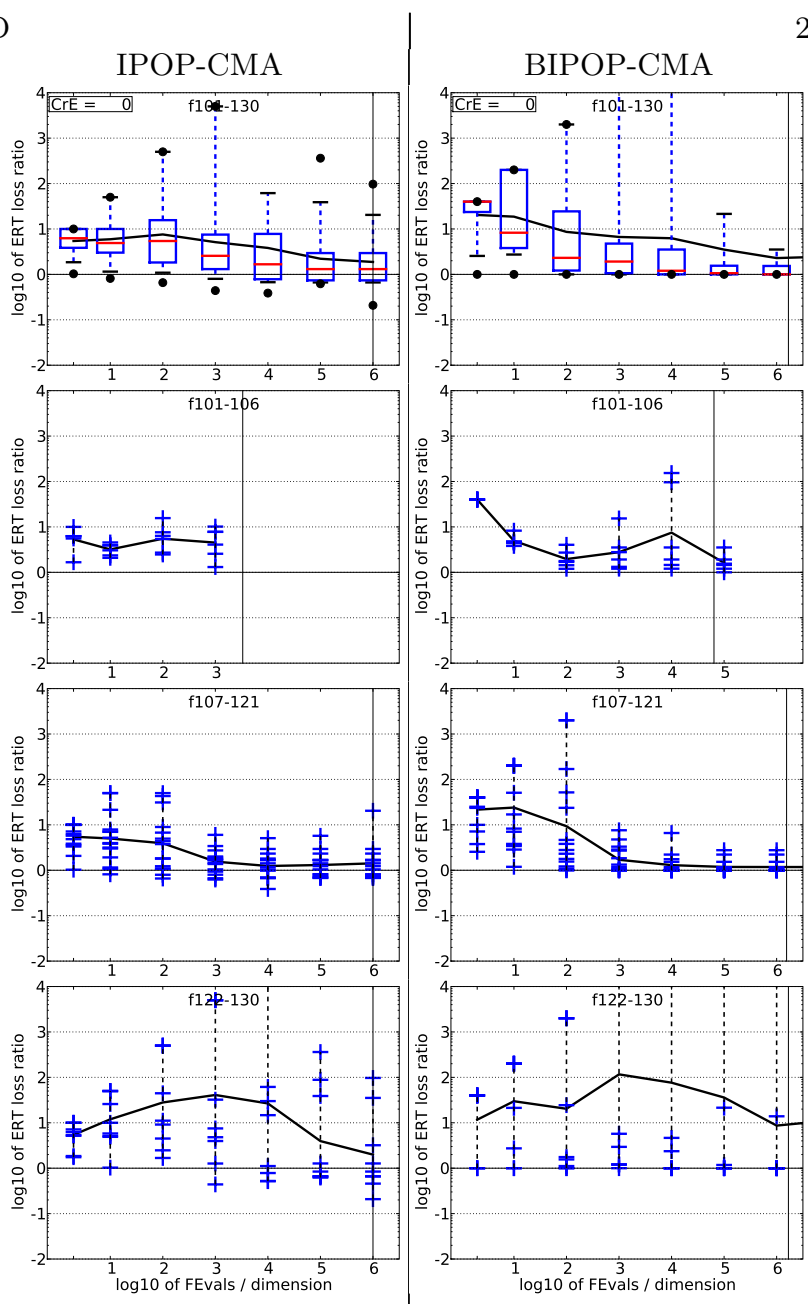

$20-\mathrm{D}$

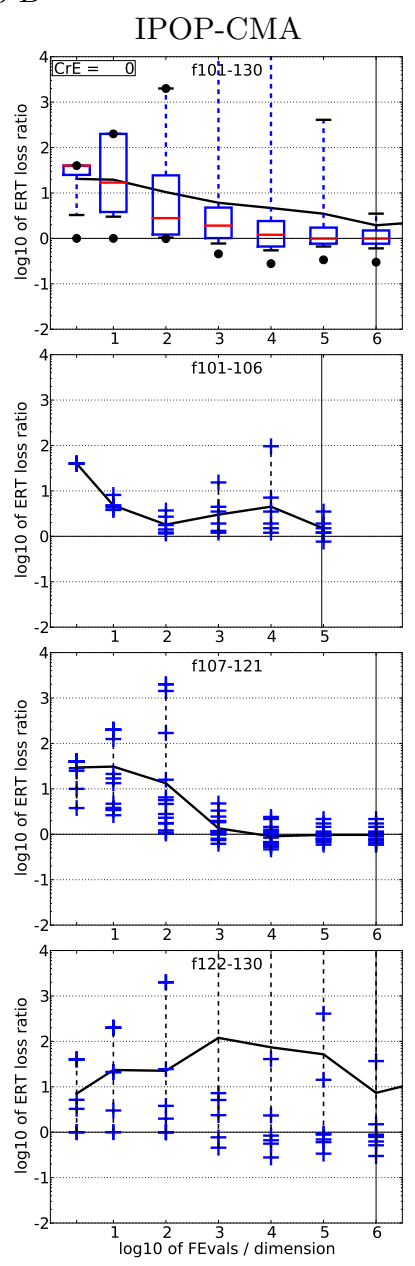

Figure 4: ERT loss ratio versus given budget FEvals. The target value $f_{\mathrm{t}}$ for ERT is the smallest (best) recorded function value such that $\operatorname{ERT}\left(f_{\mathrm{t}}\right) \leq \mathrm{FEvals}$ for the presented algorithm. Shown is FEvals divided by the respective best $\operatorname{ERT}\left(f_{\mathrm{t}}\right)$ from BBOB-2009 for functions $f_{101}-f_{130}$ in 5-D and 20-D. Each ERT is multiplied by $\exp (\mathrm{CrE})$ correcting for the parameter crafting effort. Line: geometric mean. Box-Whisker error bar: 25-75\%-ile with median (box), 10-90\%-ile (caps), and minimum and maximum ERT loss ratio (points). The vertical line gives the maximal number of function evaluations in this function subset.

2010: Presentation of the noisy functions. Technical Report 2009/21, Research Center PPE, 2010.

[3] N. Hansen. The CMA evolution strategy: a comparing review. In J. Lozano, P. Larranaga, I. Inza, and E. Bengoetxea, editors, Towards a new evolutionary computation. Advances on estimation of distribution algorithms, pages 75-102. Springer, 2006.

[4] N. Hansen. Benchmarking a bi-population CMA-ES on the BBOB-2009 noisy testbed. In F. Rothlauf, editor, GECCO (Companion), pages 2397-2402. ACM, 2009.

[5] N. Hansen, A. Auger, S. Finck, and R. Ros.

Real-parameter black-box optimization benchmarking 2010: Experimental setup. Technical Report RR-7215, INRIA, 2010.

[6] N. Hansen, S. Finck, R. Ros, and A. Auger.

Real-parameter black-box optimization benchmarking 2009: Noisy functions definitions. Technical Report RR-6869, INRIA, 2009. Updated February 2010.
[7] N. Hansen and S. Kern. Evaluating the CMA evolution strategy on multimodal test functions. In X. Yao et al., editors, Parallel Problem Solving from Nature - PPSN VIII, LNCS 3242, pages 282-291. Springer, 2004

[8] N. Hansen, A. Niederberger, L. Guzzella, and P. Koumoutsakos. A method for handling uncertainty in evolutionary optimization with an application to feedback control of combustion. IEEE Transactions on Evolutionary Computation, 13(1):180-197, 2009.

[9] N. Hansen and A. Ostermeier. Completely derandomized self-adaptation in evolution strategies. Evolutionary Computation, 9(2):159-195, 2001.

[10] K. Price. Differential evolution vs. the functions of the second ICEO. In Proceedings of the IEEE International Congress on Evolutionary Computation, pages 153-157, 1997. 
Table 2: ERT loss ratio (see Figure 4) compared to the respective best result from BBOB-2009 for budgets given in the first column. The last row $R L_{U S} / D$ gives the number of function evaluations in unsuccessful runs divided by dimension. Shown are the smallest, $10 \%$-ile, $25 \%$-ile, $50 \%$-ile, $75 \%$-ile and $90 \%$-ile value (smaller values are better). ERT Loss ratio is equal to zero if the algorithm considered outperformed all algorithms from BBOB-2009.

\begin{tabular}{|c|c|c|c|c|c|c|c|c|c|c|c|c|c|}
\hline & & & BIP & $\mathrm{OP}-\mathrm{CM}$ & & & & & $\mathrm{POP}-\mathrm{Cl}$ & & & & \\
\hline & $f_{101}$ & $1-f 13$ & in 5 - & $\mathbf{D}, \max$ & $\mathrm{FE} / \mathrm{D}=$ & $2.23 \mathrm{e} 6$ & & $f_{10}$ & $-f_{130}$ & in $5-D$ & $\operatorname{maxF}$ & $\mathrm{D} / \mathrm{D}=1$ & $00 \mathrm{e} 6$ \\
\hline \#FEs/D & best & $10 \%$ & $25 \%$ & med & $75 \%$ & $90 \%$ & \#FEs/D & best & $10 \%$ & $25 \%$ & med & $75 \%$ & $90 \%$ \\
\hline 2 & 1.3 & 2.1 & 4.5 & 7.3 & 10 & 10 & 2 & 1.0 & 1.8 & 3.8 & 6.2 & 10 & 10 \\
\hline 10 & 1.3 & 1.5 & 2.6 & 4.3 & 13 & 39 & 10 & 0.81 & 1.1 & 3.0 & 4.7 & 10 & 50 \\
\hline 100 & 1.0 & 1.1 & 2.4 & 4.9 & 16 & $2.7 \mathrm{e} 2$ & 100 & 0.66 & 1.1 & 1.8 & 5.2 & 16 & $2.8 \mathrm{e} 2$ \\
\hline $1 \mathrm{e} 3$ & 1.0 & 1.0 & 1.3 & 1.8 & 7.8 & $2.5 \mathrm{e} 3$ & $1 \mathrm{e} 3$ & 0.44 & 0.73 & 1.3 & 2.3 & 7.5 & $2.5 \mathrm{e} 3$ \\
\hline $1 \mathrm{e} 4$ & 1.0 & 1.0 & 1.0 & 1.7 & 7.2 & $2.5 \mathrm{e} 4$ & $1 \mathrm{e} 4$ & 0.39 & 0.60 & 0.78 & 1.5 & 7.8 & 46 \\
\hline $1 \mathrm{e} 5$ & 1.0 & 1.0 & 1.0 & 1.7 & 3.5 & 9.2 & $1 \mathrm{e} 5$ & 0.62 & 0.66 & 0.74 & 1.3 & 2.9 & 25 \\
\hline $1 \mathrm{e} 6$ & 1.0 & 1.0 & 1.0 & 1.7 & 2.1 & 6.5 & $1 \mathrm{e} 6$ & 0.21 & 0.66 & 0.74 & 1.3 & 2.9 & 15 \\
\hline $\mathrm{RL}_{\mathrm{US}} / \mathrm{D}$ & $8 \mathrm{e} 5$ & $8 \mathrm{e} 5$ & $9 \mathrm{e} 5$ & $1 \mathrm{e} 6$ & $1 \mathrm{e} 6$ & $1 \mathrm{e} 6$ & $\mathrm{RL}_{\mathrm{US}} / \mathrm{D}$ & $4 \mathrm{e} 5$ & $4 \mathrm{e} 5$ & $5 \mathrm{e} 5$ & $8 \mathrm{e} 5$ & $1 \mathrm{e} 6$ & $1 \mathrm{e} 6$ \\
\hline & f $101^{-}$ & $f 130$ & in 20 & $\begin{array}{l}\mathrm{OP}-\mathrm{CM} \\
\mathrm{D}, \max \end{array}$ & $\mathrm{FE} / \mathrm{D}=$ & $1.68 \mathrm{e} 6$ & & $f_{101}$ & $\begin{array}{l}\mathrm{POP}-\mathrm{C} \\
-\boldsymbol{f} \mathbf{1 3 0}\end{array}$ & $-\infty$ & & C/D= & $00 \mathrm{e} 6$ \\
\hline \#FEs/D & best & $10 \%$ & $25 \%$ & med & $75 \%$ & $90 \%$ & $\# \mathrm{FEs} / \mathrm{D}$ & best & $10 \%$ & $25 \%$ & med & $75 \%$ & $90 \%$ \\
\hline 2 & 1.0 & 1.8 & 24 & 40 & 40 & 40 & 2 & 1.0 & 2.1 & 25 & 40 & 40 & 40 \\
\hline 10 & 1.0 & 2.0 & 3.8 & 8.3 & $2.0 \mathrm{e} 2$ & $2.0 \mathrm{e} 2$ & 10 & 1.0 & 2.8 & 3.8 & 15 & $2.0 \mathrm{e} 2$ & $2.0 \mathrm{e} 2$ \\
\hline 100 & 1.0 & 1.0 & 1.2 & 2.0 & 24 & $2.0 \mathrm{e} 3$ & 100 & 0.99 & 1.0 & 1.2 & 2.8 & 24 & $2.0 \mathrm{e} 3$ \\
\hline $1 \mathrm{e} 3$ & 1.0 & 1.0 & 1.1 & 1.9 & 4.8 & $2.0 \mathrm{e} 4$ & $1 \mathrm{e} 3$ & 0.46 & 0.76 & 1.0 & 1.9 & 4.8 & $2.0 \mathrm{e} 4$ \\
\hline $1 \mathrm{e} 4$ & 1.0 & 1.0 & 1.0 & 1.2 & 3.5 & $1.0 \mathrm{e} 5$ & $1 \mathrm{e} 4$ & 0.28 & 0.54 & 0.66 & 1.2 & 2.4 & $1.0 \mathrm{e} 5$ \\
\hline $1 \mathrm{e} 5$ & 1.0 & 1.0 & 1.0 & 1.0 & 1.5 & 12 & $1 \mathrm{e} 5$ & 0.34 & 0.63 & 0.76 & 0.96 & 1.7 & $2.1 \mathrm{e} 2$ \\
\hline $1 \mathrm{e} 6$ & 1.0 & 1.0 & 1.0 & 1.0 & 1.5 & 3.1 & $1 \mathrm{e} 6$ & 0.30 & 0.60 & 0.76 & 0.96 & 1.5 & 2.8 \\
\hline $1 \mathrm{e} 7$ & 1.0 & 1.0 & 1.0 & 1.0 & 1.5 & 3.1 & $1 \mathrm{e} 7$ & 0.52 & 0.63 & 0.77 & 1.1 & 1.7 & 3.7 \\
\hline $\mathrm{RL}_{\mathrm{US}} / \mathrm{D}$ & $4 \mathrm{e} 5$ & $5 \mathrm{e} 5$ & $1 \mathrm{e} 6$ & $1 \mathrm{e} 6$ & $1 \mathrm{e} 6$ & $1 \mathrm{e} 6$ & $\mathrm{RL}_{\mathrm{US}} / \mathrm{D}$ & $1 \mathrm{e} 5$ & $2 \mathrm{e} 5$ & $6 \mathrm{e} 5$ & $9 \mathrm{e} 5$ & $1 \mathrm{e} 6$ & $1 \mathrm{e} 6$ \\
\hline
\end{tabular}

